\title{
Successful Empiric Therapy for Postsplenectomy Sepsis with Campylobacter fetus in an Abattoir Worker with Follicular Lymphoma
}

\author{
Hideyuki Nakazawa ${ }^{1,2}$, Sayaka Nishina ${ }^{1,2}$, Hitoshi Sakai ${ }^{1,2}$, Toshiro Ito ${ }^{2}$, \\ Fumihiro Ishida ${ }^{1,3}$ and Kiyoshi Kitano ${ }^{2}$
}

\begin{abstract}
:
Asplenia may yield an increased risk of fulminant sepsis with various pathogens. Human infection with Campylobacter fetus is rare, but it often presents with non-gastrointestinal tract infection among immunocompromised individuals. A 55-year-old abattoir worker presented with a fever. He had had splenectomy for follicular lymphoma and rituximab maintenance therapy by four months before the presentation. Blood cultures yielded $C$. fetus, and the administration of meropenem dissolved the bacteremia. Further maintenance therapy was withheld, and no recurrence of infection has been observed for seven years. Asplenia, occupational exposure, and/or rituximab maintenance therapy might have been precipitating factors of this rare infection.
\end{abstract}

Key words: Campylobacter fetus, sepsis, splenectomy, rituximab, follicular lymphoma, abattoir

(Intern Med 57: 3329-3332, 2018)

(DOI: 10.2169/internalmedicine.1031-18)

\section{Introduction}

The asplenic population is heterogeneous and includes patients with splenectomy after trauma, hematologic diseases such as malignant lymphoma or sickle cell disease, chronic idiopathic thrombocytopenic purpura or chronic graft-versushost disease after allogeneic hematopoietic stem cell transplantation, untreated human immunodeficiency virus infection, or severe celiac disease $(1,2)$. While the risk of postsplenectomy sepsis is highly variable according to the underlying causes of asplenia as well as the patient's age or the interval since splenectomy, its mortality and morbidity are generally high (1). Overwhelming postsplenectomy infection (OPSI), an extremely severe case, is defined as septicemia and/or meningitis occurring at any time after splenectomy and carries a very high mortality of $50 \%$ (3). The most common pathogens of postsplenectomy sepsis are encapsulated organisms, represented by Streptococcus pneumoniae and Haemophilus influenza type b. Guidelines recommend vaccinations to reduce the risk of invasive pneumo- coccal disease substantially (4). An increased risk of infection in those with asplenia has also been reported with Neisseria meningitides, Capnocytophaga canimorsus, $C$. cynodegmi, Babesia microti, Plasmodium species, Bordetella holmesii, Escherichia coli, Staphylococcus suis and $S$. aureus $(1,5,6)$.

Campylobacter are Gram-negative zoonotic bacterial pathogens causing human and animal diseases. $C$. jejuni and C. coli account for 95\% of human Campylobacter isolates, and their infections predominantly cause diarrheal disease. C. fetus, on the other hand, is frequently responsible for non-gastrointestinal tract infection, such as sepsis, endocarditis, infectious aneurysms, osteomyelitis, and meningitis (7). Human infection of $C$. fetus is rather rare, and it is more likely to be developed among immunocompromised hosts (7). Anecdotes and small case series have clarified that hematological diseases may also pose an increased risk of infection with $C$. fetus $(8,9)$.

We herein report a case of successful empiric antimicrobial therapy for postsplenectomy sepsis with $C$. fetus. Since postsplenectomy sepsis with this organism has been scarcely

${ }^{1}$ Division of Hematology, Department of Internal Medicine, Shinshu University School of Medicine, Japan, ${ }^{2}$ Department of Hematology, NHO Matsumoto Medical Center, Japan and ${ }^{3}$ Biomedical Laboratory Sciences, Shinshu University School of Medicine, Japan Received: February 19, 2018; Accepted: April 17, 2018; Advance Publication by J-STAGE: July 6, 2018 Correspondence to Dr. Hideyuki Nakazawa, hnaka@shinshu-u.ac.jp 
Table. Laboratory Data at Admission.

\begin{tabular}{lclllc}
\hline WBC & $14.6 \times 10^{3} / \mu \mathrm{L}$ & total protein & $5.8 \mathrm{~g} / \mathrm{dL}$ & uric nitrogen & $33 \mathrm{mg} / \mathrm{dL}$ \\
myelocyte & $2.0 \%$ & albumin & $2.8 \mathrm{~g} / \mathrm{dL}$ & creatinine & $1.48 \mathrm{mg} / \mathrm{dL}$ \\
metamyelocyte & $2.0 \%$ & total bilirubin & $1.0 \mathrm{mg} / \mathrm{dL}$ & $\mathrm{Na}$ & $132 \mathrm{mmol} / \mathrm{L}$ \\
stab & $7.0 \%$ & ALP & $636 \mathrm{U} / \mathrm{L}$ & $\mathrm{K}$ & $4.1 \mathrm{mmol} / \mathrm{L}$ \\
segmented & $87.0 \%$ & $\gamma \mathrm{GTP}$ & $69 \mathrm{U} / \mathrm{L}$ & $\mathrm{Cl}$ & $93 \mathrm{mmol} / \mathrm{L}$ \\
lymphocyte & $2.0 \%$ & AST & $37 \mathrm{U} / \mathrm{L}$ & $\mathrm{CRP}$ & $37.83 \mathrm{mg} / \mathrm{dL}$ \\
hemoglobin & $12.7 \mathrm{~g} / \mathrm{dL}$ & ALT & $44 \mathrm{U} / \mathrm{L}$ & $\mathrm{IgG}$ & $946 \mathrm{mg} / \mathrm{dL}$ \\
MCV & $90.3 \mathrm{fL}$ & LDH & $175 \mathrm{U} / \mathrm{L}$ & $\mathrm{IgA}$ & $111 \mathrm{mg} / \mathrm{dL}$ \\
platelet & $15.2 \times 10^{4} / \mu \mathrm{L}$ & amylase & $22 \mathrm{U} / \mathrm{L}$ & IgM & $66 \mathrm{mg} / \mathrm{dL}$ \\
\hline
\end{tabular}

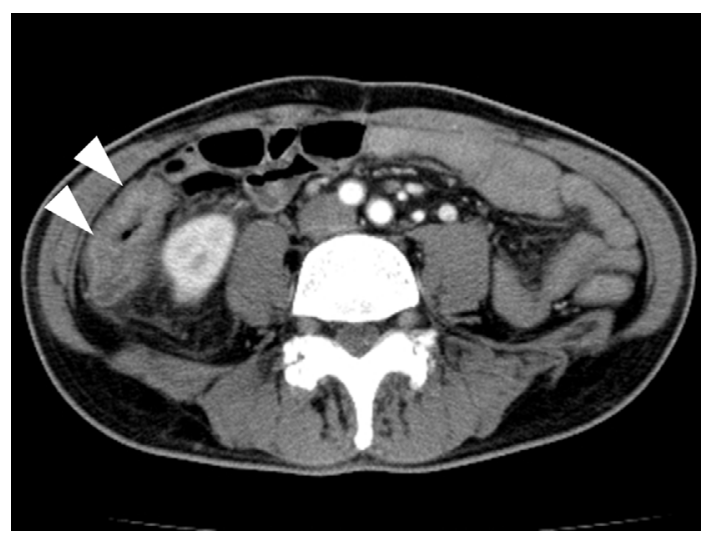

Figure. Enhanced computed tomography showed that the ascending colon (arrow heads) was edematous.

described, we discuss the risk factors and likely causes that may have contributed to this rare infection. We obtained written informed consent from the presented patient according to the Declaration of Helsinki for the current report.

\section{Case Report}

A 55-year-old male veterinarian was admitted to our hospital because of a 2-month history of an intermittent fever. About two years before the admission, he had first come to a medical attention because of an 8 -cm submucosal mass in the stomach detected at a medical checkup. Because a definitive diagnosis could not be made pre-operatively, he had undergone total gastrectomy, cholecystectomy and splenectomy for the suspected advanced malignancy at a local hospital. The surgical pathology revealed gastric follicular lymphoma, grade 2, with stage IIE disease. Two weeks after the surgery, a 23-valent pneumococcal polysaccharide vaccine was administered. Computed tomography (CT), fluorodeoxyglucose-positron emission tomography (FDGPET), and a bone marrow biopsy showed no signs of residual lymphoma.

About a year after the surgery, he requested rituximab maintenance therapy for follicular lymphoma and received $375 \mathrm{mg} / \mathrm{m}^{2} /$ week rituximab for four weeks at our hospital. Three months after the rituximab administration (approximately 1.5 years after splenectomy), he started to complain of a loss of appetite and an intermittent fever. Oral levoflox- acin was prescribed and started soon after each febrile episode. The fever was ameliorated temporarily, but another febrile episode recurred several days after the cessation of the previous prescription. With the recurrent febrile episodes for two months, he became debilitated and was admitted to our hospital. He reported no history of diarrhea. He was a veterinarian working at an abattoir and he had no other medical history beside the follicular lymphoma. His colleagues at the abattoir had not experienced any infectious episodes, as he had explained.

At admission, the patient was weary and feverish, but a physical examination did not show any local signs of infection. Given the possibility of OPSI, the empiric administration of meropenem was initiated soon after three sets of blood culture. His blood counts and chemistries revealed a significant inflammatory response (Table). His serum level of immunoglobulins was within the reference range of normal. An interferon-gamma release assay for mycobacterium was negative. A pharyngeal swab tested negative for influenza virus antigen with rapid paper chromatography. X-ray and CT showed that he did not have relapse of lymphoma nor any documented infection, such as pneumonia. The ascending colon was partially edematous (Figure), but a total colonoscopy failed to confirm any pathologic changes in the mucosal membrane. There were no peripheral lymphadenopathies. Echocardiography did not reveal any signs of endocarditis.

The blood cultures yielded Gram-negative rods, but subsequent passaged cultures were not initially successful, so the pathogens could not be identified. Feces culture isolated non-pathologic E. coli and Bacillus spp., which were considered part of his normal intestinal flora. The one-week administration of meropenem ameliorated the fever, but a week after its discontinuation, fervescence ensued. The same antibiotics were restarted after another three sets of blood culture, which produced identical Gram-negative spiral-shaped organisms. With the successful passage of cultures under 25 ${ }^{\circ} \mathrm{C}$ microaerobic conditions, $C$. fetus was identified based on its biochemical profiles as evaluated with the API-campy test kit (SYSMEX bioMérieux, Tokyo, Japan). The fourweek administration of meropenem resolved the bacteremia. Additional rituximab maintenance therapy was withheld, and he also changed his workplace from the abattoir to an office. The patient has been free from further episodes of bactere- 
mia, and there has been no recurrence of lymphoma for seven years.

\section{Discussion}

The present case exhibited a persistent infection of $C$. $f e$ tus for about two months despite intermittent antibiotic therapies. The Gram-negative organisms repeatedly detected in multiple blood cultures were retrospectively considered to have been the same bacteria. C. fetus is known to harbor surface-layer proteins (SLPs), which account for its resistance to complement-mediated killing and phagocytosis (10). $\mathrm{Tu}$ et al. observed high-frequency DNA rearrangement within the coding regions for the SLPs in as short as a 20day interval, implying that a persistent infection of $C$. fetus could be facilitated by the organism's ability to adjust its antigenicity of SLPs to the environmental challenges (10). The SLPs might therefore have been associated with the refractory nature of the infection in the present case, although a study to examine the SLP expression of the present strain could not be performed.

The spleen is a major site of immune response to bloodborne antigens, and it harbors macrophages responsible for clearing the blood of unwanted substances even in the absence of specific immunity (11). Asplenia may lead to impairment of phagocytic functions and dysfunction in both adaptive and innate immunity (5). As a result, asplenic patients may have an impaired clearance capacity of not only IgG-coated bacteria but also non-opsonized pathogens and may carry a life-long risk of infection $(1,6)$. Rituximab reduces the population of antibody-producing precursor plasma cells, leading to a decreased antigenic response. The serum complement may also be excessively consumed by the ADCC activity of rituximab in a setting of a high tumor burden (12). A meta-analysis of rituximab maintenance therapy for follicular lymphoma showed an elevated rate of infection-related adverse events, although the infections did not reduce the overall survival (13). Though the total serum gamma globulin level was not decreased after rituximab administration (data not shown), both splenectomy and adjuvant rituximab administration might have contributed to the functional impairment of the humoral responses. However, which factor contributed most to the bacteremia in the present case remains unclear.

Human infection of $C$. fetus is more likely to be observed among at-risk individuals with underlying medical conditions than in the general population. Sakran et al. described a patient with $C$. fetus infection after splenectomy for thalassemia (14). According to anecdotes or small case series, other predisposing conditions may include diabetes mellitus, HIV infection, hematological malignancy, hypogammaglobulinemia, liver cirrhosis, corticosteroid and other immunosuppressive treatments, cardiovascular disease with valve abnormalities, and medical device implants (7-9, 15-18). Aging and pregnancy may also increase the risk of its infection $(7,19)$. Wagenaar et al. suggested that the general population might be repeatedly exposed to undetected levels of C. fetus through contaminated foods or other routes (7), implying the importance of a high level of caution among high-risk individuals.

In the present case of asplenia, environmental exposure to the pathogen may have been an additional risk factor. Outbreaks of $C$. fetus infection as an occupational hazard among abattoir workers and farmers without known underlying medical conditions have been previously described $(20,21)$. The abnormal ascending colon detected on CT might have been a route of infection after the oral exposure to $C$. fetus at the abattoir. However, similar infectious episodes among our patient's coworkers at his work place were not noted. His underlying medical conditions, including asplenia and rituximab exposure, might therefore have been more strongly associated with the increased risk of $C$. fetus infection than excessive exposure through his working environment, although occupational surveillance was not conducted for this pathogen.

Prophylactic antimicrobial therapy after splenectomy is recommended to prevent fulminant sepsis for only select patients, such as children under 5 years of age (1). However, for adult patients, there is no standard antimicrobial prophylaxis, and empiric antibiotics and patient education may be more important. Since the initial symptoms of asplenic sepsis may be nonspecific and mild (22), a high level of caution and empiric intervention may be warranted to decrease its severity (6). Antimicrobial resistance among Campylobacter spp. is a worldwide phenomenon. Fernandez-Cruz et al. mention that quinolones should be discouraged for empirical therapy for the bacteremia (23), while Liao et al. assert that carbapenems should be considered (24). Susceptibility test results of the strain for CFPM, MEPM, and MINO in the present case were 'resistant', 'susceptible', and 'susceptible', respectively. Although the antibiotic selection should be guided by the susceptibility of the individual strain, the optimum choice and duration of empiric therapy for at-risk individuals remain controversial.

The present case may therefore be a representative case of an at-risk asplenic individual, as this patient might have harbored an immunocompromised medical condition and been subject to an at-risk work environment. The present findings should encourage medical staff to discuss the risks of lesscommon infections with asplenic patients.

\section{The authors state that they have no Conflict of Interest (COI).}

\section{References}

1. Rubin LG, Schaffner W. Clinical practice. Care of the asplenic patient. N Engl J Med 371: 349-356, 2014.

2. Nakazawa H, Makishima H, Ito T, et al. Screening tests using serum tissue transglutaminase $\operatorname{IgA}$ may facilitate the identification of undiagnosed celiac disease among Japanese population. Int J Med Sci 11: 819-823, 2014.

3. Waghorn DJ. Overwhelming infection in asplenic patients: current best practice preventive measures are not being followed. J Clin 
Pathol 54: 214-218, 2001.

4. Moberley S, Holden J, Tatham DP, Andrews RM. Vaccines for preventing pneumococcal infection in adults. Cochrane Database Syst Rev Cd000422, 2013.

5. O'Neal HR Jr, Niven AS, Karam GH. Critical illness in patients with asplenia. Chest 150: 1394-1402, 2016.

6. Spelman D, Buttery J, Daley A, et al. Guidelines for the prevention of sepsis in asplenic and hyposplenic patients. Intern Med J 38: 349-356, 2008.

7. Wagenaar JA, van Bergen MA, Blaser MJ, Tauxe RV, Newell DG, van Putten JP. Campylobacter fetus infections in humans: exposure and disease. Clin Infect Dis 58: 1579-1586, 2014.

8. Chuman Y, Takata T, Sameshima H, et al. Campylobacter fetus bacteremia in a patient with adult $\mathrm{T}$ cell leukemia. Clin Infect Dis 36: 1497-1498, 2003

9. Yamagami K, Miyashita T, Nakamura T, et al. Campylobacter fetus bacteremia with purulent pleurisy in a young adult with primary hypogammaglobulinemia. Intern Med 53: 1221-1225, 2014.

10. Tu ZC, Gaudreau C, Blaser MJ. Mechanisms underlying Campylobacter fetus pathogenesis in humans: surface-layer protein variation in relapsing infections. J Infect Dis 191: 2082-2089, 2005.

11. Kipps TJ. The organization and structure of lymphoid tissues. In: Williams Hematology. Kaushansky K, Beutler E, Kipps TJ, Seligsohn U, Prchal JT, Eds. McGrawHill, New York, 2010: 75-84.

12. Kennedy AD, Beum PV, Solga MD, et al. Rituximab infusion promotes rapid complement depletion and acute CD20 loss in chronic lymphocytic leukemia. J Immunol 172: 3280-3288, 2004.

13. Vidal L, Gafter-Gvili A, Salles G, et al. Rituximab maintenance for the treatment of patients with follicular lymphoma: an updated systematic review and meta-analysis of randomized trials. J Natl Cancer Inst 103: 1799-1806, 2011.

14. Sakran W, Raz R, Levi Y, Colodner R, Koren A. Campylobacter bacteremia and pneumonia in two splenectomized patients. Eur $\mathrm{J}$ Clin Microbiol Infect Dis 18: 496-498, 1999.
15. Krutchik AN, Velasquez W. Campylobachter fetus infection in a patient with Hodgkin's disease. JAMA 238: 1810-1811, 1977.

16. Gazaigne L, Legrand P, Renaud B, et al. Campylobacter fetus bloodstream infection: risk factors and clinical features. Eur J Clin Microbiol Infect Dis 27: 185-189, 2008.

17. Ahmar W, Johnson D, Richards M, Strathmore N. Campylobacter fetus infection of an internal cardioverter defibrillator. Pacing Clin Electrophysiol 31: 258-259, 2008.

18. Miki K, Maekura R, Hiraga T, et al. Infective tricuspid valve endocarditis with pulmonary emboli caused by Campylobacter fetus after tooth extraction. Intern Med 44: 1055-1059, 2005.

19. Zonios DI, Panayiotakopoulos GD, Kabletsas EO, Tzima EL, Stefanou I, Archimandritis AJ. Campylobacter fetus bacteraemia in a healthy individual: clinical and therapeutical implications. J Infect 51: 329-332, 2005.

20. de Perio MA, Niemeier RT, Levine SJ, Gruszynski K, Gibbins JD. Campylobacter infection in poultry-processing workers, Virginia, USA, 2008-2011. Emerg Infect Dis 19: 286-288, 2013.

21. Ellis A, Irwin R, Hockin J, Borczyk A, Woodward D, Johnson W. Outbreak of Campylobacter infection among farm workers: an occupational hazard. Can Commun Dis Rep 21: 153-156, 1995.

22. Stryker RM, Orton DW. Overwhelming postsplenectomy infection. Ann Emerg Med 17: 161-164, 1988.

23. Fernandez-Cruz A, Munoz P, Mohedano R, et al. Campylobacter bacteremia: clinical characteristics, incidence, and outcome over 23 years. Medicine 89: 319-330, 2010.

24. Liao CH, Chuang CY, Huang YT, Lee PI, Hsueh PR. Bacteremia caused by antimicrobial resistant Campylobacter species at a medical center in Taiwan, 1998-2008. J Infect 65: 392-399, 2012.

The Internal Medicine is an Open Access journal distributed under the Creative Commons Attribution-NonCommercial-NoDerivatives 4.0 International License. To view the details of this license, please visit (https://creativecommons.org/licenses/ by-nc-nd/4.0/).

(C) 2018 The Japanese Society of Internal Medicine Intern Med 57: 3329-3332, 2018 